\title{
Ulnar neuropathy due to heterotopic ossification: A rare complication after stroke
}

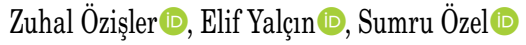 \\ Department of Physical Medicine and Rehabilitation, Ministry of Health Ankara Physical Medicine and Rehabilitation Training and Research Hospital, Ankara, Turkey
}

Received: January 26, 2016 Accepted: April 12, 2016 Published online: May 07, 2018

\begin{abstract}
Heterotopic ossification (HO) is a rare complication (0.5 to 1.2\%) after stroke. Although there are few reports on ulnar neuropathy (UN) due to $\mathrm{HO}$ at the elbow after traumatic brain injury, thermal burn or upper limb trauma, there has been no case reported after stroke. Herein, we present a 32-year-old male patient with UN due to HO after stroke.
\end{abstract}

Keywords: Elbow; heterotopic ossification; stroke; ulnar neuropathy.

The upper extremity complications after stroke can adversely affect the process and outcomes of the rehabilitation. ${ }^{[1]}$ Compared to other upper extremity complications, heterotopic ossification $(\mathrm{HO})$ is extremely rare (0.5 to $1.2 \%)$ after stroke. ${ }^{[2]}$

Heterotopic ossification is usually diagnosed about four months after stroke. The initial symptoms of $\mathrm{HO}$ are often loss of joint mobility and pain. In the late stages, ectopic bone can compress vascular and neurogenic structures. ${ }^{[3]}$ The ulnar nerve is the most susceptible neurovascular structure to compression associated with $\mathrm{HO}$ at the elbow. Although there are few reports on ulnar neuropathy (UN) due to $\mathrm{HO}$ at the elbow after traumatic brain injury, upper extremity trauma (i.e., fracture, dislocation), and thermal burn, there has been no case reported after stroke. ${ }^{[4-6]}$

Herein, we present a hemiplegic patient with UN due to $\mathrm{HO}$ at the elbow to draw attention to the factors which may affect the outcomes of stroke rehabilitation.

\section{CASE REPORT}

A 32-year-old male patient presented with rightsided hemiplegia following a subarachnoid hemorrhage due to arteriovenous malformation one year ago. The patient had a history of one month stay in the intensive care unit. The range of motion exercises were started at the first month and the patient was admitted in our clinic for stroke rehabilitation. A written informed consent was obtained from the patient.

The patient was independently ambulated with a tripod. The functional ambulation scale was assessed as Level 4. The Brunnstrom scale was Stage V for the right upper extremity, IV for the right hand, and $\mathrm{V}$ for the right lower extremity. There was 45 degrees flexion contracture in the right elbow (Figure 1). There was no limitation in the supination and pronation of the forearm. Deep tendon reflexes of the right upper extremity were hyperactive. There were atrophies on the both thenar and hypothenar eminences. The Benediction posture was seen at his right hand (Figure 2). Hypoesthesia was present on volar and dorsal aspects of the fourth and fifth finger. Loss of thumb adduction was present resulting in positive Froment sign. Loss of finger abduction and adduction was present in the right hand. There was also weakness in the distal interphalangeal joint flexion of the fourth and fifth finger.

Radiographic imaging showed heterotopic bone formation in the soft tissues surrounding the right elbow (Figure 3). Motor and sensory conduction studies of the ulnar, median, radial and dorsal ulnar cutaneous 


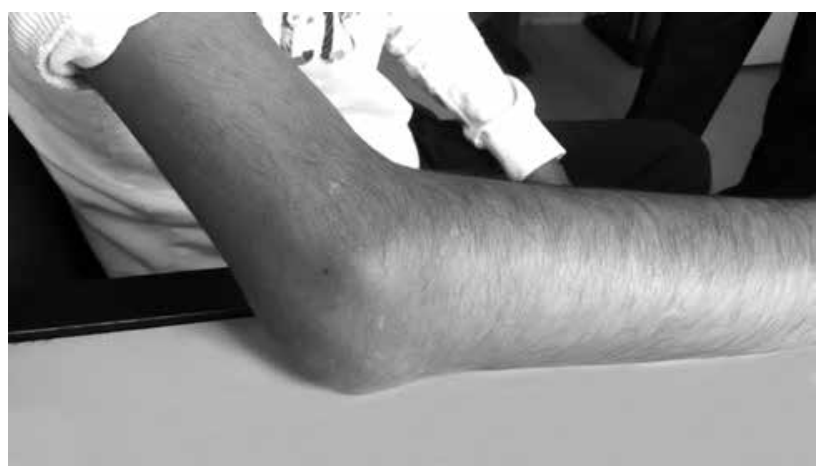

Figure 1. The position of the patient's elbow.

(DUC) nerves of right hand and electromyography (EMG) of the abductor digiti minimi (ADM), flexor carpi ulnaris (FCU), flexor digitorum profundus (FDP), abductor pollicis brevis (APB), extensor indicis proprius (EIP), cervical $8(\mathrm{C} 8)$ and thoracal $1\left(\mathrm{~T}_{1}\right)$ paraspinal muscles were performed to exclude lower brachial plexopathy or C8-T1 radiculopathy.

Compound muscle action potentials (CMAPs) were recorded from the ADM by surface electrode in response to the stimulation at the wrist, below elbow, and above elbow. The CMAPs amplitude $(0.2 \mathrm{mV}$ - the lower limit of ulnar CMAP is $8.7 \mathrm{mV}$ according to our EMG laboratory) were significantly decreased at all site of stimulations. Sensory nerve action potentials of the right ulnar nerve from the fifth finger and right DUC nerve were unable to be obtained. Electromyography of the right ADM, FCU, FDP showed severe reduction in voluntary recruitment with fibrillation potentials and positive sharp waves. There was no abnormality in

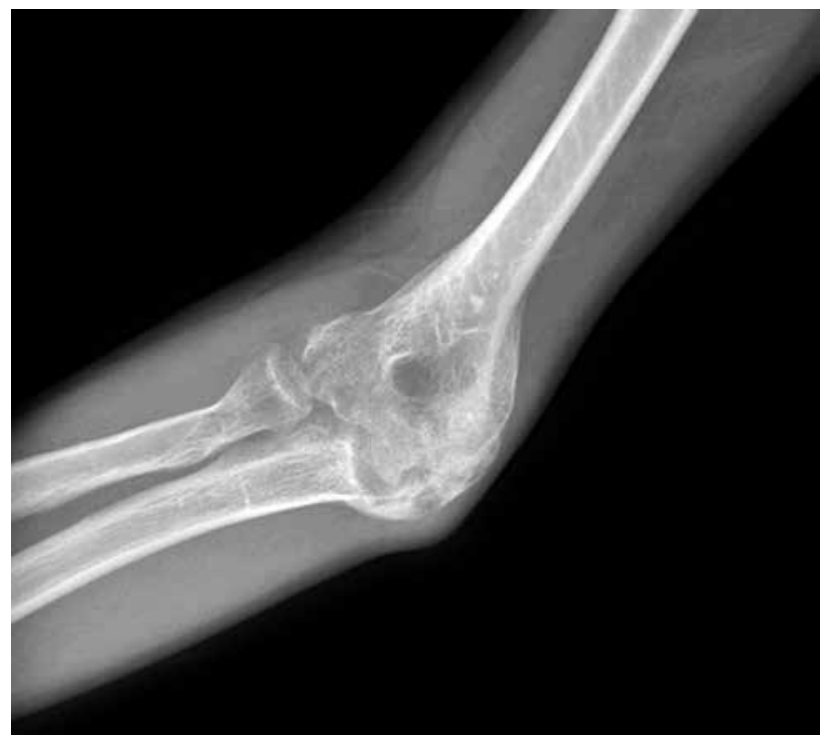

Figure 3. The X-ray image of the patient's right elbow.

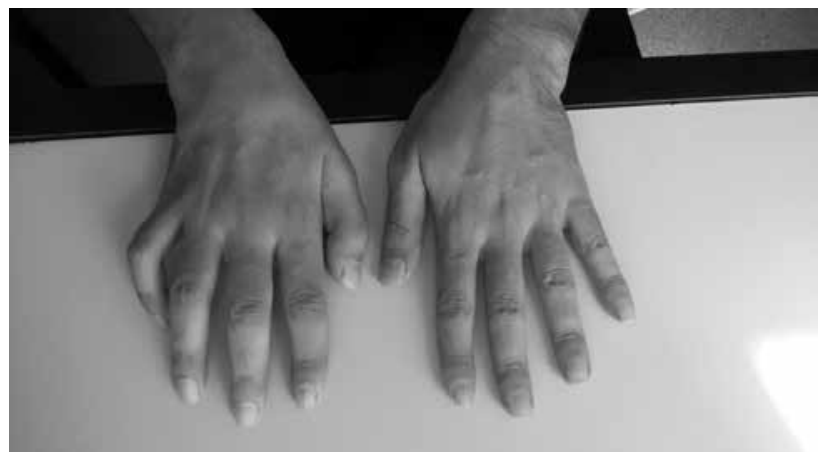

Figure 2. The position of the patient's hand.

the motor and sensory conduction studies of the right median and radial nerves. Electromyography of APB, EIP, and C8-T1 paraspinal muscles were normal. These results were compatible with ulnar nerve entrapment around the right elbow with axonal loss, as right DUC nerve conductions were unable to be obtained and spontaneous activities were seen at EMG of the right FCU and FDP which muscular branches are exited at the elbow level.

Ultrasonographic evaluation of the ulnar nerve throughout the right upper extremity (starting from the axilla until the wrist) was done using a linear array probe (7-12 MHz Logiq P5a). All the measurements were performed in axial views. The right ulnar nerve was observed expanded at the level of the right retro epicondylar groove $\left(14.2 \mathrm{~mm}^{2}\right)$ (Figure 4). The ultrasonographic evaluation also revealed accompanying heterotopic bony spurs located very proximity to the right ulnar nerve.

The patient underwent range of motion exercises, gentle stretching, and occupational therapy in our

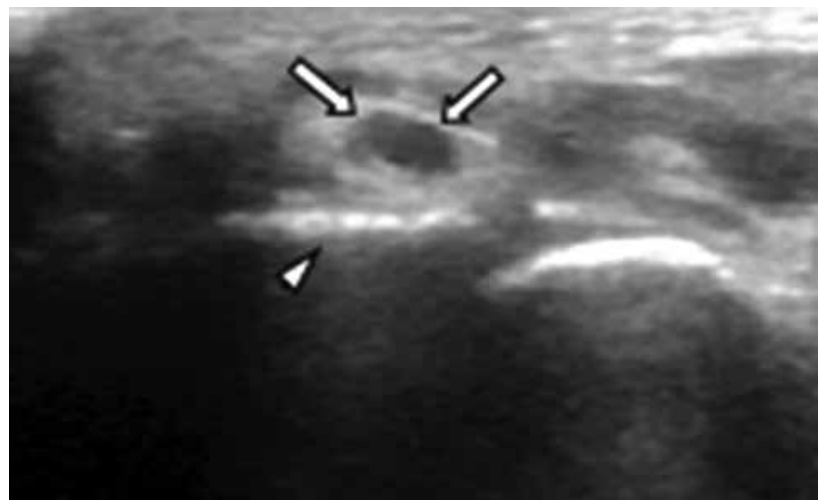

Figure 4. Ultrasonographic imaging (axial view) of right cubital tunnel demonstrating enlargement of the ulnar nerve (arrows) with irregular bony spurs (arrow head). 
clinic. Pregabalin $(300 \mathrm{mg} / \mathrm{day})$ were administered for neuropathic pain. The upper limb function of the patient was evaluated with quick Disabilities of the Arm, Shoulder, and Hand (DASH) after rehabilitation program and his total score was 74 (The quick DASH scaled on a 0-100 scale. A higher score indicates greater disability).

\section{DISCUSSION}

Although upper extremity complications are common problems after stroke, $\mathrm{HO}$ is a rare complication. It can affect large joints on the hemiparetic side of the body, particularly in patients who have some coexisting traumatic injury. ${ }^{[1]}$ The incidence of $\mathrm{HO}$ at the elbow after fracture is 1.9 to $3 \%$ in the literature. ${ }^{[7,8]}$ However, there is no data about the incidence of $\mathrm{HO}$ at the elbow after stroke. ${ }^{[2]}$ Heterotopic ossification can affect the upper limb function due to limitation of range of motion and compression to adjacent neurovascular structures ${ }^{[3]}$ In this report, $\mathrm{UN}$ associated with elbow $\mathrm{HO}$ is presented. Although a few cases of UN associated $\mathrm{HO}$ at the elbow have been reported after brain injury, thermal burn, and upper limb trauma, there is no case report after stroke. ${ }^{[4-6,9]}$ Heterotopic ossification may have negative implications on the rehabilitation process and outcomes. In our case, upper limb functions were impaired due to limited range of motion, pain and UN, which have a negative impact on self-care activities. Limitation of the elbow motion led to difficulties in bathing and hair care, and while UN caused loss of hand grip.

While EMG is useful in determining both the level and severity of neuropathy, ultrasonography is very practical in evaluating the anatomical relation of the nerve. Ultrasonographic evaluation of our patient demonstrated that $\mathrm{HO}$ was very close to the ulnar nerve, but there was no direct compression to the nerve. Although $\mathrm{HO}$ usually does not cause direct compression, contracture of the elbow may cause traction of the nerve and space narrowing may lead to secondary compression to the ulnar nerve. ${ }^{[6,10,1]]}$

In conclusion, we present a case of a rare condition in stroke and discuss the importance of the factors affecting the rehabilitation process. If a patient with stroke presents with atrophy, sensory and motor deficits which are incompatible with hemiplegic severity, the presence of an accompanying neuropathy should be considered. Electromyography and ultrasonography are very useful diagnostic methods.

\section{Declaration of conflicting interests}

The authors declared no conflicts of interest with respect to the authorship and/or publication of this article.

\section{Funding}

The authors received no financial support for the research and/or authorship of this article.

\section{REFERENCES}

1. Harvey RL, Roth EJ, Yu DT, Celnik P. Stroke syndromes. In: Braddom RL, editor. Physical Medicine and Rehabilitation: Philadelphia: Elsevier; 2011. p. 1177-222.

2. Pek CH, Lim MC, Yong R, Wong HP. Neurogenic heterotopic ossification after a stroke: diagnostic and radiological challenges. Singapore Med J 2014;55:119-22.

3. Vanden Bossche L, Vanderstraeten G. Heterotopic ossification: a review. J Rehabil Med 2005;37:129-36.

4. Hamdi MF, Aloui I, Allagui M. Ulnar nerve compression at the elbow and heterotopic ossification: a report of five cases. Neurochirurgie 2010;56:340-3.

5. Fikry T, Saidi H, Madhar M, Latifi M, Essadki B. Cubital tunnel syndrome and heterotopic ossification. Eight case reports. Chir Main 2004;23:109-13.

6. Yang SC, Chen AC, Chao EK, Yuan LJ, Lee MS, Ueng SW. Early surgical management for heterotopic ossification about the elbow presenting as limited range of motion associated with ulnar neuropathy. Chang Gung Med J 2002;25:245-52.

7. Josefsson PO, Johnell O, Gentz CF. Long-term sequelae of simple dislocation of the elbow. J Bone Joint Surg Am 1984;66:927-30.

8. Summerfield SL, DiGiovanni C, Weiss AP. Heterotopic ossification of the elbow. J Shoulder Elbow Surg 1997;6:32132.

9. Wainapel SF, Rao PU, Schepsis AA. Ulnar nerve compression by heterotopic ossification in a head-injured patient. Arch Phys Med Rehabil 1985;66:512-4.

10. Apfelberg DB, Larson SJ. Dynamic anatomy of the ulnar nerve at the elbow. Plast Reconstr Surg 1973;51:79-81.

11. Kara M, Ekiz T, Öztürk GT, Onat ŞŞ, Özçakar L. Heterotopic Ossification and Peripheral Nerve Entrapment: Ultrasound is a Must-use Imaging Modality. Pain Med 2015;16:1643-4. 